\title{
Period-Doubling Analysis and Chaos Detection Using Commercial Harmonic Balance Simulators
}

\author{
Juan-Mari Collantes, Member, IEEE, and Almudena Suárez, Member, IEEE
}

\begin{abstract}
Two of the most common phenomena leading to chaos are the period-doubling cascade and the formation of transverse homoclinic orbits. In this paper, a bifurcation analysis technique is presented for the prediction of both phenomena in microwave circuits. The fact that the technique is based on the use of commercial harmonic balance software constitutes a major advantage for the circuit designer. The accuracy of the method relies on the capability to detect and calculate the successive period doublings, which, in period-doubling cascades, provides a good estimation of the parameter values for the onset of chaos. Another important aspect of the new method is the equilibrium point determination, necessary for the prediction of the homoclinic chaos. The accuracy in the calculation of the limit cycle, taking into account the most influential period doublings, ensures a good estimation of the parameter values for the formation of possible homoclinic orbits. In order to validate the method, it is initially applied to an $R L$-diode circuit, with a period-doubling route to chaos. A practical microwave frequency doubler is then analyzed, determining its parameter ranges for stable operation. Excellent results are obtained in comparison with the time-domain simulations. As an example of the method's capabilities for the prediction of homoclinic chaos, the bifurcation loci of Chua's circuit, with a cubic nonlinearity, are obtained and they agree closely with time-domain simulations.
\end{abstract}

Index Terms-Chaos, frequency division, harmonic balance, stability.

\section{INTRODUCTION}

$\mathbf{T}$ HE two most important characteristics of chaotic responses in nonlinear circuits are the sensitive dependence on the initial conditions and the continuity of the spectra [1]. This kind of behavior is often encountered in microwave circuits of autonomous nature, such as oscillators or frequency dividers. Due to the spectrum-continuity characteristic, chaos is often identified with an anomalous increase of the noise level at the measurement stage.

Although important applications have recently been found for chaotic synchronization [2], [3], chaotic responses are usually undesirable for the designer of microwave components. Therefore, chaos prediction at the simulation stage may be invaluable in reducing the development cycles of monolithic microwave integrated circuits (MMIC's). Due to the continuity of the spectra, frequency-domain techniques, such as harmonic

Manuscript received January 5, 1999. This work was supported by the Caja de Ahorros de Santander y Cantabria, and by the University of the Basque Country under Research Project UPV 224.310-EA051/98.

J.-M. Collantes is with the Electricity and Electronics Department, University of the Basque Country, 48080 Bilbao, Spain (e-mail: jmcollan@we.lc.ehu.es).

A. Suárez is with the Department Ingeniería de Comunicaciones, Universidad de Cantabria, 39005 Santander, Spain (e-mail: almu@dicom.unican.es).

Publisher Item Identifier S 0018-9480(00)02783-6. balance (HB), cannot be used for the analysis of steady-state chaotic responses. On the other hand, the long transients with respect to the signal period of microwave circuits generally prevent time-domain analysis. However, the main interest of the circuit designer is not the accurate determination of the chaotic solutions, but its prediction. Therefore, a good strategy may be the detection of possible bifurcations leading to chaos from steady-state regimes, determinable through HB. In this paper, an HB technique is proposed for the detection of two different phenomena often accompanying chaotic behavior: the formation of transverse homoclinic orbits [4], [5] and the period-doubling cascade [5], [6].

In the period-doubling cascade to chaos, the period of the circuit solution is successively doubled as a parameter is varied, obtaining frequency divisions by two, four, eight, 16 , etc. The length of the parameter intervals for the successive frequency divisions decreases at a rate that rapidly approaches the Feigenbaum number 4.67 , chaos being obtained when the period grows ad infinitum [5]. In Section II-A, the period-doubling cascade to chaos is presented in more detail.

Homoclinic orbits are formed when the stable and unstable manifolds of a saddle equilibrium point (EP) intersect. When this intersection is transversal, it gives rise to a chaotic solution. This solution is often found when a limit cycle (LC) collides with a saddle EP after some period-doubling bifurcations [7]. Transversal homoclinic orbits are a common cause of chaos in circuits in which the current-voltage characteristics are $N$-shaped [8], like Gunn and tunnel diode oscillators. In Section II-B, homoclinic chaos is presented in more detail.

In the period-doubling cascade, there is an inherent need for the prediction and calculation of the successive period-doubled responses. This is not a simple task when using frequency-domain techniques such as the HB method. Due to its forced nature [9], HB fails to initialize all frequency components apart from the external generator frequencies and their spectral components, even when the Fourier frequency basis is properly supplied. This is unfortunately the case of the subharmonic frequencies, at which no generators are present. In order to cope with this difficulty, a new technique is proposed here, based on the use of auxiliary generators (AG's) at the subharmonic frequencies. These generators, introduced only for simulation purposes, make the HB operate in its natural forced way and guarantee the convergence toward the subharmonic solutions. The advantage of the AG's is that they can be easily used with commercial HB simulators, as has been done here, by simply introducing them into the circuit schematic. This enables the analysis of frequency divisions and other complex analysis, otherwise impossible in commercial simulators. In period-doubling cascades, 
the parameter accumulation properties [5], together with the capability to determine the initial frequency divisions, enable the estimation of the parameter values for the onset of chaos. The new technique for the period-doubling analysis and its application to the prediction of chaos are presented in Section III-A.

The method proposed here for the prediction of homoclinic chaos is based on the detection of the collision between the LC and saddle EP. It is a multiharmonic generalization of the one presented by Genesio [7] and based on the use of the describing function. Here, the determination and analysis of the circuit EP's are carried out through a very simple technique. In the new method, it has also been considered that the good accuracy in the prediction of the onset of chaos is closely related to the accuracy in the LC determination. For that, a realistic calculation of the waveform, considering the initial and most influential frequency divisions, is required. Thus, the technique developed here for the calculation of the successive period doublings turns out to be crucial. The entire new method for the detection of homoclinic chaos will be presented in Section III-B.

In order to validate the new analysis method, several circuits have been simulated, using commercial HB software. The first application is an $R L$-diode frequency divider, exhibiting a period doubling route to chaos [13]. This circuit has been analyzed, estimating the parameter values for the onset of chaotic behavior. A microwave frequency doubler, with a similar period-doubling cascade, has also been analyzed, determining its parameter ranges for stable behavior. For homoclinic chaos prediction, the well-known Chua's circuit [4]-[10], with a cubic nonlinearity, has been chosen, as it represents a paradigm for the study of chaotic behavior. The details and results of each application are shown in Section IV.

\section{Two COMmOn PATterns For ChaOtic Behavior}

\section{A. Period Doubling Route to Chaos}

In the period doubling route to chaos [5], [6], for some values of a parameter $\xi$, the circuit exhibits a periodic behavior of period $T$. For a critical value $\xi_{1}$, the orbit of period $T$ loses stability, creating an orbit of period $2 T$. This new periodicity remains until a new critical parameter value $\xi_{2}$ is reached, for which the period-2 solution loses stability, giving rise to a period-4 orbit. This process of successive period doubling continues, with decreasing ranges $\left(\xi_{n}, \xi_{n+1}\right)$ of $2^{n} T$ period, until, at a certain parameter value $\xi_{\infty}$, the period doubles ad infinitum, obtaining a chaotic behavior [5], [6].

Let $\Delta_{n}$ be the parameter interval $\left(\xi_{n}, \xi_{n+1}\right)$ for frequency division by $2^{n}$. Feigenbaum [5] has shown that the compression rate of the successive intervals $\delta_{n}=\Delta_{n} / \Delta_{n+1}$ converges fast to $\delta=4.6692$ (Feigenbaum number).

\section{B. Homoclinic Chaos}

The constant (dc) solutions of a nonlinear circuit are given by the EP's of the nonlinear differential equations describing its behavior. When linearizing these equations about a saddle EP, the resulting eigenvalues will have negative and positive real parts [5], respectively, associated with the stable and unstable manifolds (nonlinear analogs of the eigenspaces).

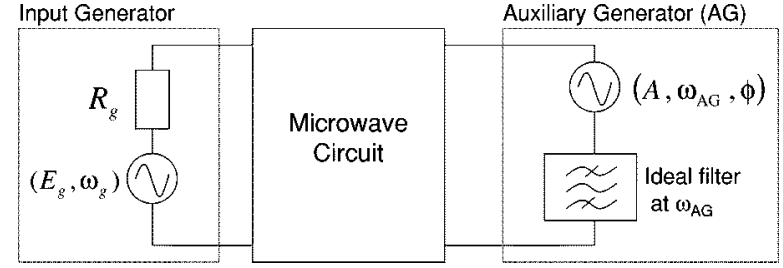

Fig. 1. AG $A, \omega_{\mathrm{AG}}$, and $\phi$ are, respectively, the amplitude, frequency, and phase of the AG.

Under some circumstances, the stable and unstable manifolds may intersect, forming what is known as a homoclinic orbit. When the homoclinic orbit is transverse (transverse intersection of the manifolds), it creates a homoclinic tangle [6]. This is a complex recurring structure, with embedded Smale horseshoes in the Poincare map [5]. The stretching and folding action of this map has a well-known relationship with chaotic behavior [6].

For the existence of horseshoes [6], some conditions must be satisfied by the eigenvalues associated to the saddle EP through which the homoclinic orbit is formed [4]. According to the theory of Sil'nikov [4], in a three-dimensional system, this point must have a pair of complex eigenvalues $-\alpha \pm j \beta$ and a real eigenvalue $\lambda$, satisfying $\alpha, \lambda>0$, and $\lambda / \alpha>1$.

When a parameter varies, homoclinic orbits are often formed when an LC collides with a saddle EP. When preceded by period-doubling bifurcations, this interaction between the $\mathrm{LC}$ and the EP is likely to give rise to a homoclinic tangle and, thus, to a chaotic behavior [7]-[10].

\section{Chaos Detection Through HB}

\section{A. Analysis of the Period-Doubling Route to Chaos}

As stated in Section I, HB has a well-known difficulty in dealing with frequency division, due to the absence of generators at the subharmonic frequencies. In order to cope with these HB difficulties, a new technique is proposed here, based on the use of AG's. These are introduced into the circuit only for simulation purposes [9]. An AG is an ideal generator followed by an ideal bandpass filter. Voltage generators are used here, although a dual analysis based on current generators is also possible. The voltage $\mathrm{AG}$ is connected in parallel at a sensitive location of the circuit (Fig. 1). The filter exhibits a zero impedance value at the generator operating frequency $\omega_{\mathrm{AG}}$ and an infinite impedance value at all the other frequencies [9]. The calculation of the initial period doublings is carried out through the following steps.

1) Detection of a Period Doubling from a Period-1 Steady State: The stability analysis of a regime of period $T$, in the presence of period-doubling perturbations, is carried out by introducing a period-2 $\mathrm{AG}$, with a negligible amplitude $A_{2}=\varepsilon$ and variable phase $\phi_{2}$, as shown in Fig. 2(a). This is equivalent to a small perturbation at the divided frequency. Let $Y_{2}$ be the input admittance observed by the AG. The period- 1 regime will be unstable if the conditions for the division startup $\operatorname{Re}\left(Y_{2}\right)<$ $0, \operatorname{Im}\left(Y_{2}\right)=0$ are satisfied for a certain phase value of the AG. This may be easily determined in commercial software by sweeping the AG phase $\phi_{2}$ between $0^{\circ}-180^{\circ}$.

2) Determination of the Period-2 Steady State: For obtaining the period-2 steady state, the amplitude $A_{2}$ and phase 

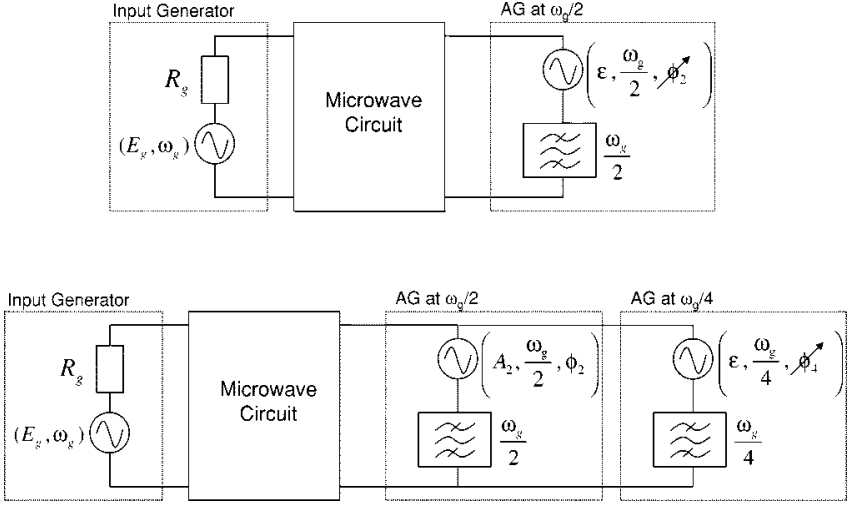

Fig. 2. (a) Connection of one AG for the prediction of a period-2 solution. Negligible amplitude $A_{2}=\varepsilon$ and variable phase $\phi_{2}$. (b) Connection of two AG for the prediction of a period-4 solution. $A_{2}$ and $\phi_{2}$ are maintained at their steady-state values. Negligible amplitude $A_{4}=\varepsilon$ and variable phase $\phi_{4}$.

$\phi_{2}$ of the AG are optimized, using the commercial software tools, in order to obtain a zero value of the observed admittance magnitude $\left|Y_{2}\right|$. When the condition $\left|Y_{2}\right|=0$ is fulfilled, the steady state is attained and the AG does not perturb the circuit solution. Note that the inclusion of this AG resolves the HB difficulties with subharmonic frequencies.

3) Detection of a Period Doubling from a Period-2 Steady State: In order to predict a new possible period doubling, the period-2 AG must be kept at its steady-state value. An additional AG of period $4 T$ is introduced, with a negligible amplitude $A_{4}=\varepsilon$ and a variable phase $\phi_{4}$, as shown in Fig. 2(b). A sweep in $\phi_{4}$, between $0^{\circ}-90^{\circ}$, is now carried out calculating the observed admittance $Y_{4}$. A new period doubling is obtained when the start-up conditions $\operatorname{Re}\left(Y_{4}\right)<0, \operatorname{Im}\left(Y_{4}\right)=0$ are fulfilled.

4) Determination of the Period-4 Steady State: For obtaining the period- 4 steady state, an optimization is performed in the two AG's, with the four optimization variables $A_{2}, \phi_{2}$, $A_{4}$, and $\phi_{4}$. The optimization goal will now be the zero value of the two admittance amplitudes $\left|Y_{2}\right|=0$ and $\left|Y_{4}\right|=0$. Again, when this condition happens, the two AG's do not perturb the steady-state solution. Note that the limited number of optimization variables and optimization goals ensures the efficiency of the optimization process.

A new period doubling (frequency division by eight) can be predicted by introducing a period- 8 AG with a negligible amplitude, proceeding in a similar way to those discussed in Sections III-A.1 and III-A.3. The parameter accumulation properties shown by Feigenbaum make the prediction of higher order divisions unnecessary in practice, and the parameter values for the onset of chaos may be estimated after only a few bifurcations.

\section{B. Analysis of Homoclinic Chaos}

For the detection of homoclinic chaos, a four-step technique is used: determination of the circuit EP's, analysis of the freerunning oscillation, analysis of the frequency divisions of lower orders, and determination of the parameter values for the interaction between the saddle EP and LC (EP-LC).

1) Determination of the Circuit EP's: The availability of an efficient tool for the determination of multiple EP's is of
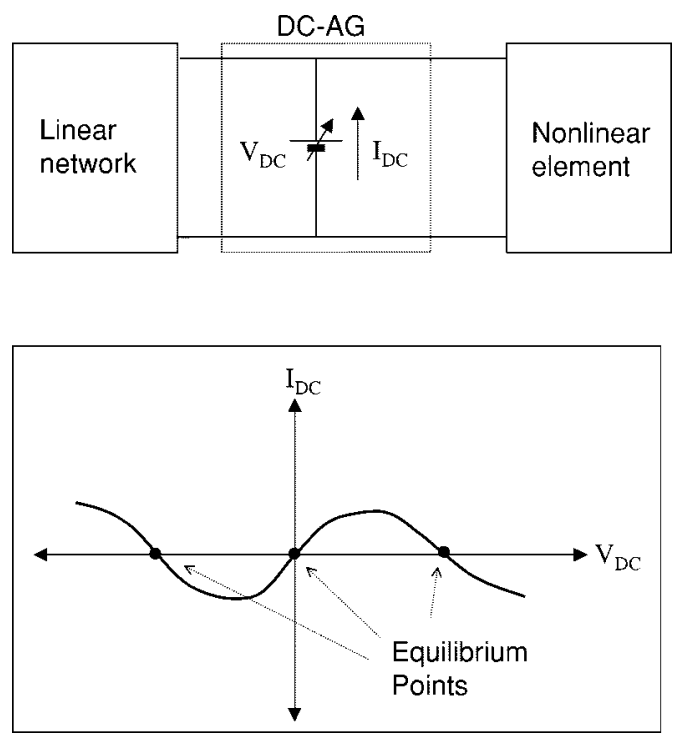

Fig. 3. Calculation of the circuit EP's. (a) DC-AG and (b) current flowing through the dc-AG as a function of the dc voltage sweep. The voltage values for which $I_{\mathrm{DC}}=0$ are the EP's.

major importance in the analysis of homoclinic chaos due to its characteristic EP-LC interaction. Here, a specific technique has been developed, very well suited for commercial simulators. It is based on the use of an auxiliary dc voltage generator (dc-AG), which is connected in parallel with the nonlinear element, as shown in Fig. 3(a). A wide range sweep is performed in the dc-AG voltage, with the EP's being given by the voltage values for which the dc current $I_{\mathrm{DC}}$ flowing through the dc-AG is equal to zero. This is shown in Fig. 3(b). Note that for zero current value, the dc-AG has no influence over the circuit solution.

For a more rigorous analysis, the stability of the different EP's must be determined, and the Nyquist stability plot, implemented in most commercial HB simulators, may be used for this purpose. Since the dc-AG is nonperturbing at the EP's, it may be kept for this analysis, which ensures the commercial simulator convergence toward the desired EP.

The Nyquist plot corresponding to an EP with a real unstable eigenvalue encircles the origin in the clockwise sense, intersecting the negative real axis of the complex plane for zero perturbation frequency [11]. If the EP has two unstable complex-conjugate eigenvalues, the locus encircles the origin in the clockwise sense, intersecting the negative real axis for a perturbation frequency different from zero [11]. This intersection provides an estimation of the free-running oscillation frequency. When modifying a circuit parameter, a Hopf bifurcation [12] will be obtained if the Nyquist plot crosses the origin at a perturbation frequency different from zero.

2) Analysis of the Free-Running Oscillation: In a second step, the periodic solution existing for some parameter ranges must be obtained. However, for circuits with multiple EP's, the standard oscillation test in HB software may search for the oscillation conditions around an EP different from the one at which oscillations start up. This difficulty is resolved here by means of a judicious voltage shift [15] in the nonlinear function, letting the simulator carry out the oscillation analysis around its default value. The periodic solution is then correctly simulated. 


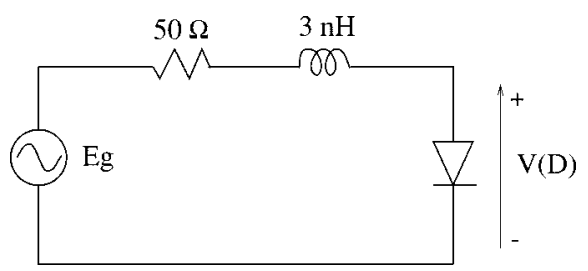

Fig. 4. Schematic of the $R L$-diode circuit. External generator frequency $f_{\text {in }}=$ $4 \mathrm{GHz}$.

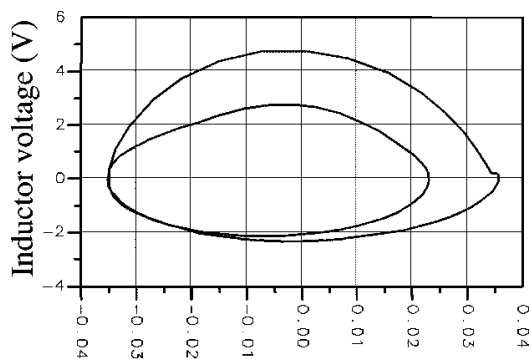

Circuit current (A)

(a)

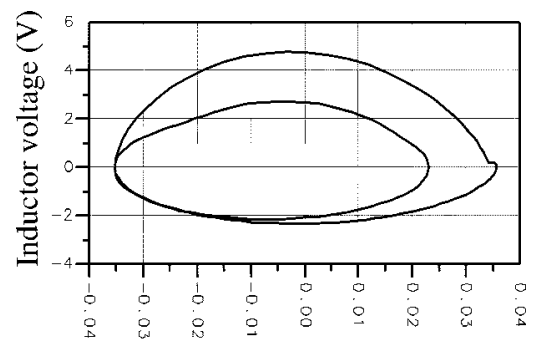

Circuit current (A)

(b)

Fig. 5. $R L$-diode circuit. Planar phase space attractor (inductor voltage versus circuit current) for $f_{\text {in }}=4 \mathrm{GHz}$ and $E g=2.5 \mathrm{~V}$ : (a) from $\mathrm{HB}$ and (b) time-domain simulations.

3) Analysis of the Frequency divisions: The third step is the prediction and calculation of the initial frequency divisions. For autonomous circuits, an extra AG, operating at the free-running amplitude and frequency, must be included. Otherwise, the HB analysis will lose its starting point. This AG is kept at its steady-state value for the first stability analysis, but will have to be optimized for the steady-state calculation of every frequency division. The calculation of these divisions is carried out by following the technique presented in Section III-A.

4) Determination of the Parameter Values for the $E P-L C$ : Finally, the fourth step checks for the possible collision between the LC and the unstable EP [7]. As will be shown, by considering a realistic waveform, including the most significant frequency divisions, the accuracy in the prediction of this collision is greatly improved in comparison with previous results [15].

\section{APPLICATION EXAMPLES}

The new method is illustrated here by means of its application to three different circuits: An $R L$-diode frequency divider and a microwave frequency multiplier, with period doubling routes to

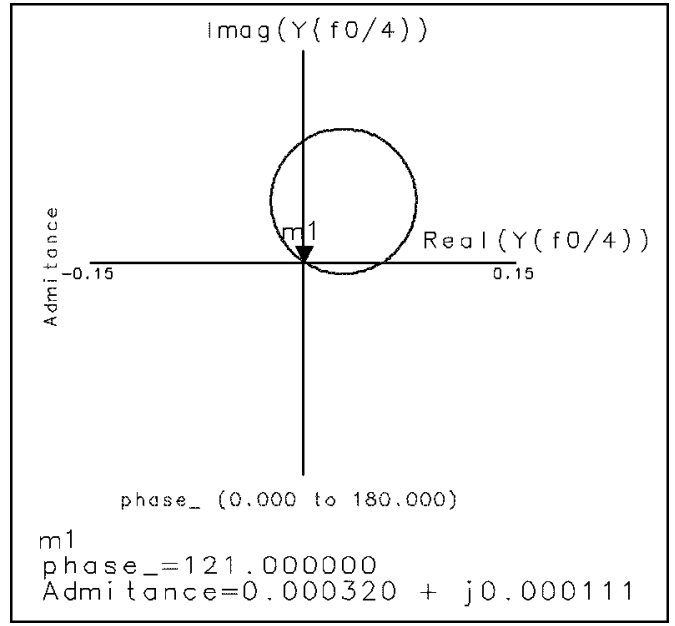

Fig. 6. $R L$-diode circuit. Prediction of the period-doubling bifurcation leading to a period-4 regime. It is obtained for $E g=4.9 \mathrm{~V}$.

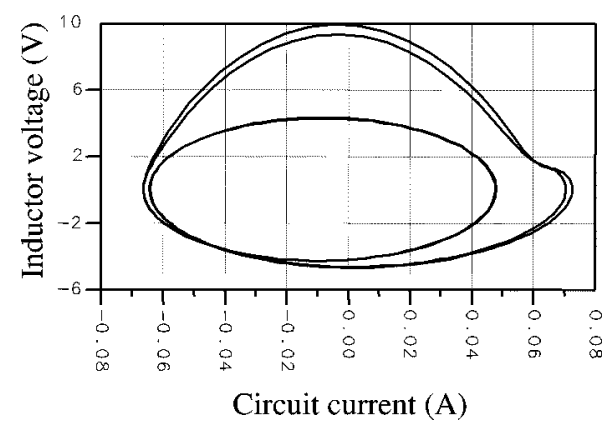

(a)

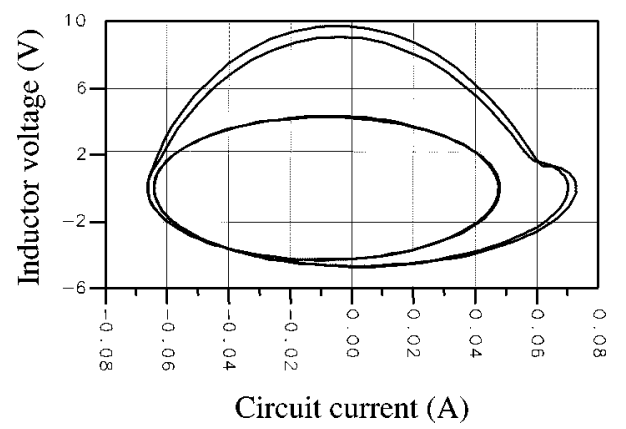

(b)

Fig. 7. $R L$-diode circuit. Planar phase space attractor (inductor voltage versus circuit current) for $f_{\text {in }}=4 \mathrm{GHz}$ and $E g=5.2 \mathrm{~V}$ : (a) from $\mathrm{HB}$ and (b) time-domain simulations.

chaos, and a Chua's circuit, with a cubic nonlinearity, exhibiting homoclinic chaos.

\section{A. RL-Diode Frequency Divider}

It has been shown [14] that varactor diodes in very nonlinear applications often show negative resistance dynamic effects, due to their high value of diffusion capacitance. Here, an $R L$-diode frequency divider [13] based on a varactor diode with a carrier lifetime $\tau=10 \mathrm{~ns}$ (Fig. 4) is analyzed. The circuit is driven by a sinusoidal voltage generator $E g$ with frequency $f_{\text {in }}=4 \mathrm{GHz}$.

The circuit stability is analyzed as a function of $E g$ by using a period-2 AG with negligible amplitude. It is possible to predict a period-doubling instability for $E g=2.1 \mathrm{~V}$. The steady 


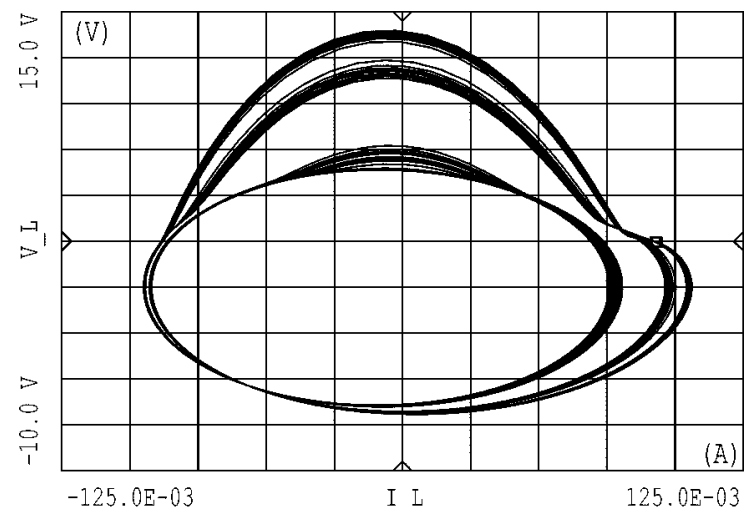

Fig. 8. $R L$-diode circuit. Time-domain chaotic attractor (inductor voltage versus circuit current) obtained for $f_{\text {in }}=4 \mathrm{GHz}$ and $E g=7.5 \mathrm{~V}$.

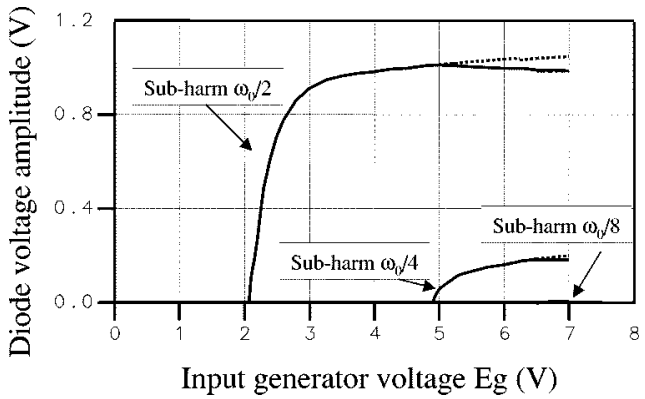

Fig. 9. $R L$-diode circuit. Bifurcation diagram as a function of the input voltage generator $E g$.

state of the period-2 solution, corresponding to $E g=2.5 \mathrm{~V}$, is calculated through $\mathrm{HB}$ by optimizing the AG. The planar phase space attractor is obtained (through inverse Fourier transform) by tracing the inductor voltage $V_{L}$ versus the current. It is shown in Fig. 5(a). The LC resulting from time-domain simulations can be observed in Fig. 5(b).

A period-4 AG, with negligible amplitude, is introduced over the steady-state period-2 solution, for the prediction of the next period doubling. When sweeping its phase, the corresponding admittance, shown in Fig. 6, crosses the origin of the polar diagram for $E g=4.9 \mathrm{~V}$, which is the bifurcation condition for the onset of the period-4 solution. The period-4 cycle obtained for $E g=5.2 \mathrm{~V}$ is shown in Fig. 7, where it can be compared with the time-domain simulation.

By introducing a period-8 AG, the onset of the period-8 solution is predicted for $E g=6.55 \mathrm{~V}$. Through time-domain simulations, the onset of chaos is obtained for $E g=6.9 \mathrm{~V}$. The time-domain chaotic attractor for $E g=7.5 \mathrm{~V}$ is shown in Fig. 8. This confirms the parameter accumulation properties of the period-doubling cascade and the method's usefulness for estimating the onset of chaos in microwave circuits.

The evolution of the circuit response as a function of the external voltage generator amplitude $E g$ can be observed in the bifurcation diagram of Fig. 9. This has been obtained in the frequency domain by tracing the amplitude of the diode voltage at the external generator frequency and at the successively generated subharmonics.

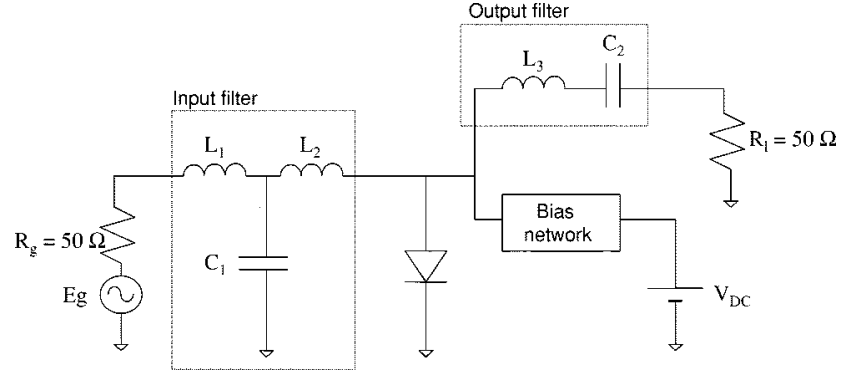

Fig. 10. Schematic of the 5-10-GHz microwave frequency doubler based on a varactor diode $\left(L_{1}=3.038 \mathrm{nH}, L_{2}=3.038 \mathrm{nH}, C_{1}=0.597 \mathrm{pF}, L_{3}=\right.$ $\left.2.533 \mathrm{nH}, C_{2}=0.1 \mathrm{pF}\right)$.

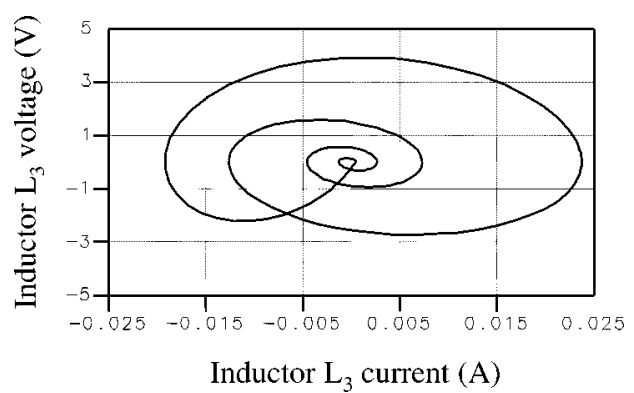

(a)

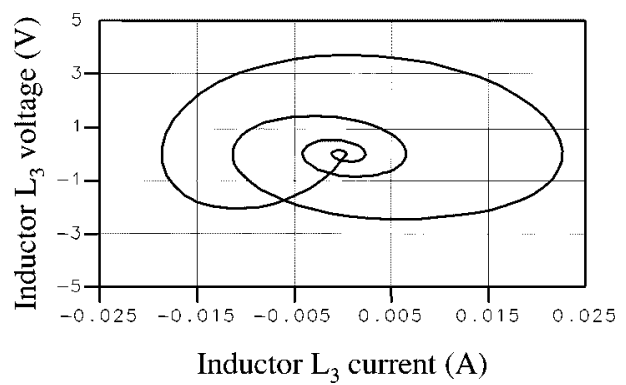

(b)

Fig. 11. Frequency doubler. Planar phase space attractor (inductor $L_{3}$ voltage versus inductor $L_{3}$ current) for $V_{\mathrm{DC}}=0 \mathrm{~V}$ and $E g=1.8 \mathrm{~V}$ : (a) from $\mathrm{HB}$ and (b) time-domain simulations.

\section{B. Microwave Frequency Doubler}

As a more practical example of the new technique for period-doubling analysis, the microwave frequency doubler of Fig. 10 is analyzed. The circuit consists of a varactor diode embedded between two filters, the input filter selecting the external generator frequency $(5 \mathrm{GHz})$ and the output filter selecting the doubled frequency $(10 \mathrm{GHz})$. The route to chaos exhibited by this kind of circuit is strongly dependent on the topology of the linear filters. For the present implementation, a period-doubling cascade is obtained, although quasi-periodic routes have also been observed for a different design of the two filters [16].

The circuit stability is analyzed as a function of the external generator voltage $E g$ and the diode bias $V_{\mathrm{DC}}$. For $V_{\mathrm{DC}}=0 \mathrm{~V}$ and $E g=1.8 \mathrm{~V}$, the circuit exhibits a period-2 solution. The planar phase space attractor is obtained here by tracing the voltage at the inductor $L_{3}$ as a function of the current through 


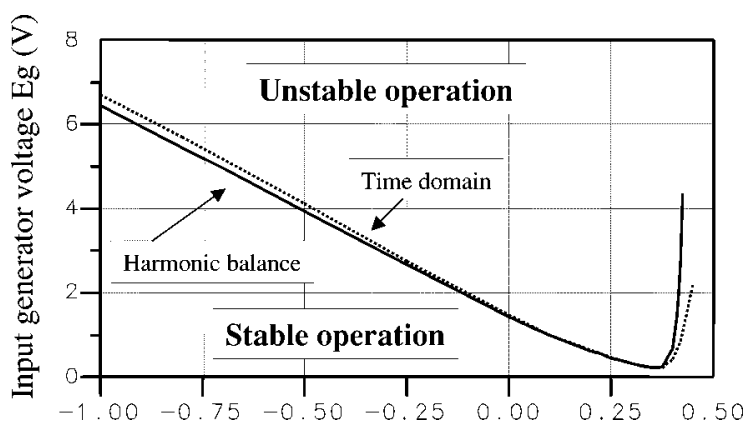

Bias voltage $\mathrm{V}_{\mathrm{DC}}(\mathrm{V})$

Fig. 12. Frequency doubler. Border for the period-2 instability as a function of $V_{\mathrm{DC}}$ and $E g$. Solid line: HB, dashed line: time-domain. The regions of stable and unstable operation as frequency doubler are also shown.

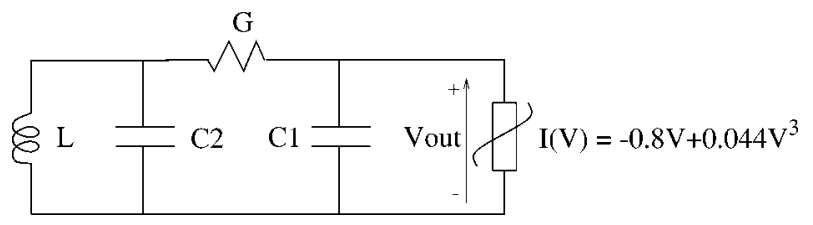

Fig. 13. Schematic of Chua's oscillator based on a cubic nonlinearity $(R=$ $\left.1.43 \Omega, L=13.6 \mathrm{pH}, C_{1}=10 \mathrm{pF}, C_{2}=0.1 \mathrm{nF}\right)$.

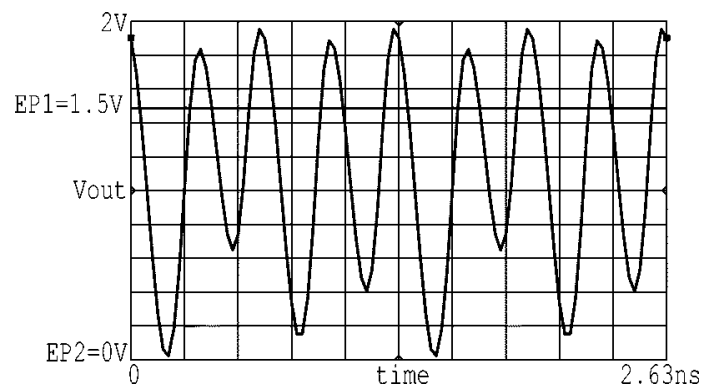

(a)

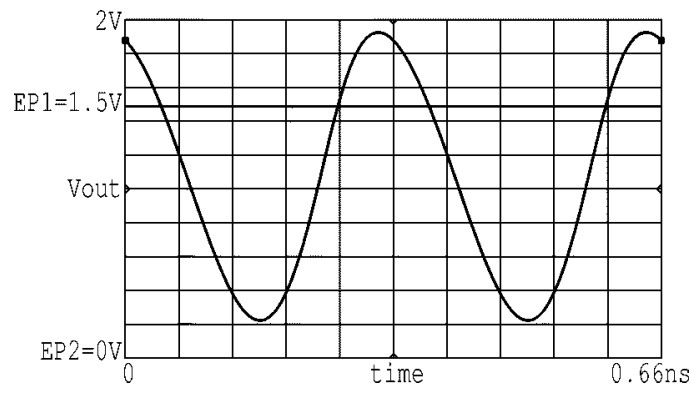

(b)

Fig. 14. Chua's oscillator with a cubic nonlinearity. (a) HB prediction of the onset of chaos from a period-4 waveform. The corresponding parameter values are $\alpha=9.1$ and $\beta=15$. (b) Waveform neglecting period doublings, obtained for the same parameter values. It is still far from the collision with the unstable $\mathrm{EP} E P_{2}=0 \mathrm{~V}$.

$L_{3}$. The comparison between the period- 2 cycle obtained from HB and time-domain simulations is shown in Fig. 11.

The border for the period-2 instability as a function of the two parameters is shown in Fig. 12, where the results from the new HB technique can be compared with those from the timedomain simulations. Thus, the new technique has enabled the determination of the input generator values for stable operation.

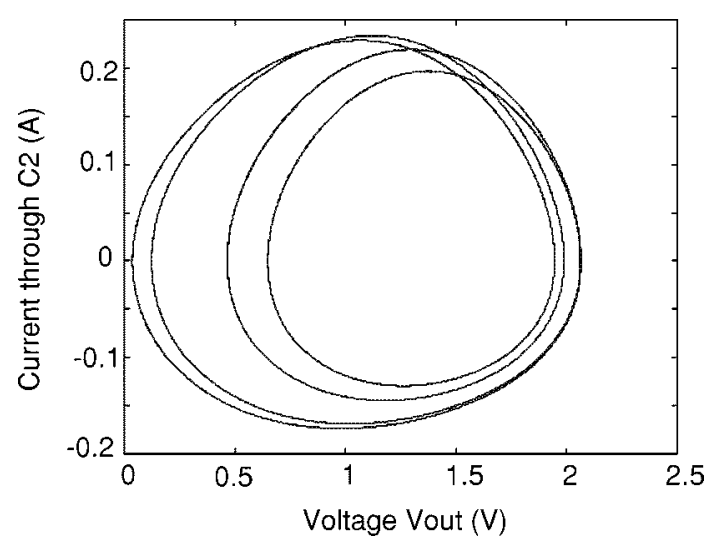

(a)

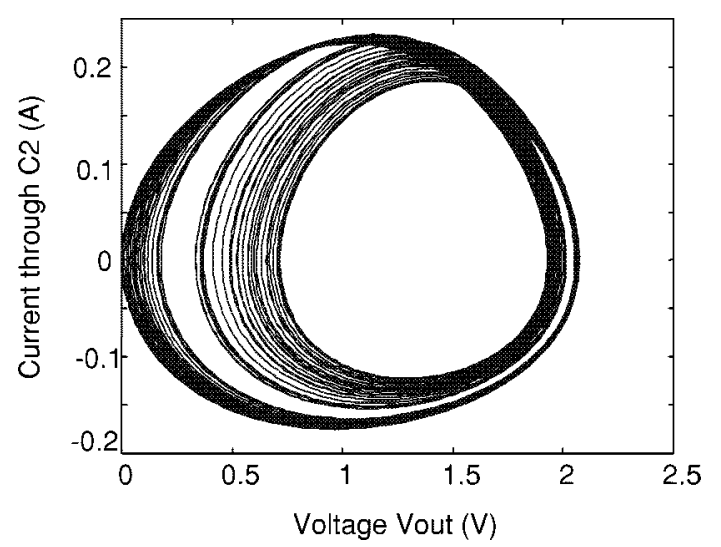

(b)

Fig. 15. Planar phase space attractors $\left(C_{2}\right.$ current versus nonlinearity voltage $)$ in Chua's oscillator with a cubic nonlinearity obtained through time-domain simulations. $\beta=15$. (a) Period-4 solution $\alpha=9.05$. (b) Chaotic attractor $\alpha=9.091$.

\section{Chua's Circuit}

A variation of Chua's circuit (Fig. 13), with a cubic nonlinearity instead of the piecewise linear resistor, has been analyzed here. Chua's circuit is commonly studied as a function of two parameters $\alpha=C_{2} / C_{1}$ and $\beta=C_{2} /\left(L G^{2}\right)$. As these parameters vary, different operating modes may be obtained, ranging from dc stable solutions to chaotic behavior.

In order to calculate the circuit EP's, a voltage dc-AG is connected in parallel with the nonlinear element. When calculating the current through this generator as a function of the corresponding voltage, three zeros are obtained, which provide the circuit EP's: $E P_{1}=1.5 \mathrm{~V}, E P_{2}=0 \mathrm{~V}$, and $E P_{3}=-1.5 \mathrm{~V}$. These values agree with the theoretical results [7].

Initially the circuit behavior is going to be analyzed for a constant $\beta=15$. The stability of the different EP's may be determined through the Nyquist plot. For $\alpha<7$, the EP $E P_{2}=0 \mathrm{~V}$ has an unstable real eigenvalue. The EP's $E P_{1}=1.5 \mathrm{~V}$ and $E P_{3}=-1.5 \mathrm{~V}$ are stable. Thus, a constant solution $V_{D}=$ $1.5 \mathrm{~V}$ or $V_{D}=-1.5 \mathrm{~V}$, with $V_{D}$ the nonlinearity voltage, is observed. This stability situation remains until the value $\alpha=7$ is reached.

For $\alpha=7$, the Nyquist stability plot corresponding to $E P_{1}=$ $1.5 \mathrm{~V}$ and $E P_{3}=-1.5 \mathrm{~V}$ crosses the origin at the perturbation frequency of $3.51 \mathrm{GHz}$, predicting a Hopf-type bifurcation. 


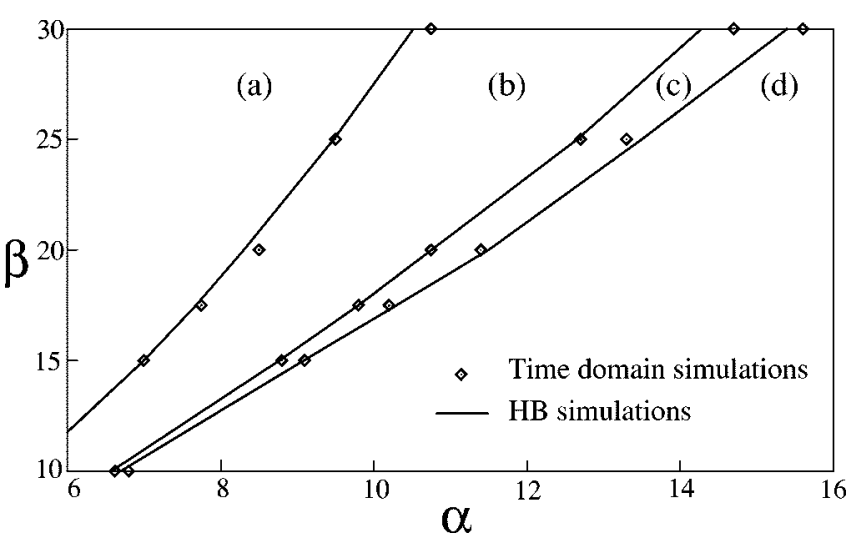

Fig. 16. Chua's oscillator with a cubic nonlinearity. Bifurcation diagramregion (a): dc stable solution, region (b): free-running oscillation, region (c): period doubling, and region (d): chaotic behavior.

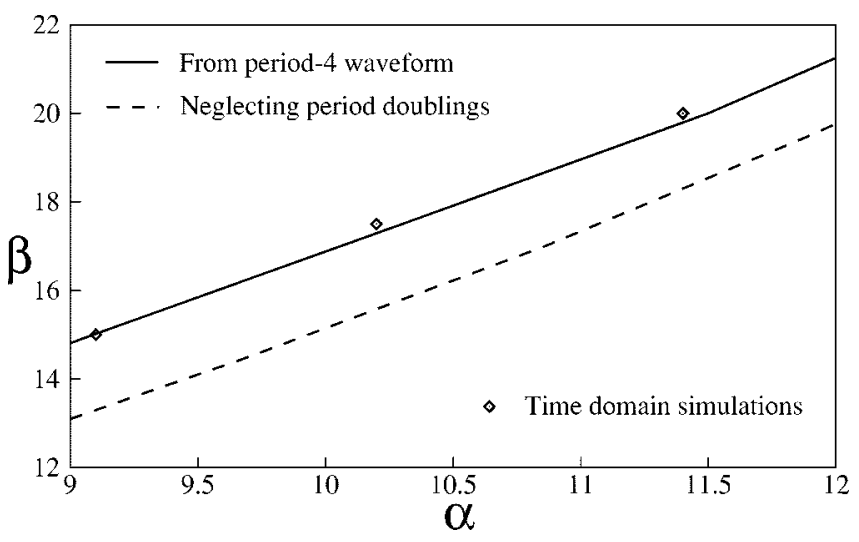

Fig. 17. Chua's oscillator with a cubic nonlinearity. Expanded bifurcation diagram. Prediction for the onset chaos when frequency divisions are neglected and when the complete period- 4 waveform is considered.

Thus, free-running oscillations are obtained around these EP's for $\alpha>7$. On the other hand, the instability of $E P_{2}=0$ remains unchanged. Through the admittance polar diagram, a period-doubling bifurcation is detected for $\alpha=8.77$. The onset of the period- 4 solution is predicted for $\alpha=9.04$.

The collision between the period- $4 \mathrm{LC}$ and the unstable EP $E P_{2}=0 \mathrm{~V}$ takes place for $\alpha=9.1$, signaling the onset of chaos. This is shown in Fig. 14(a). As shown in Fig. 14(b), for the same $\alpha$ value, when the frequency divisions are neglected [7], the LC is still far from the collision, which makes the estimated $\alpha$ value for the chaos onset higher than the real one. This error is considerably reduced when a period-four waveform is considered. Actually, there is no need to take into account period doublings of higher orders, due to the parameter accumulation properties.

In order to verify the chaos-onset prediction, time-domain simulations have been carried out for increasing $\alpha$ value. The planar phase space attractor is calculated by tracing the current through $C_{2}$ as a function of the nonlinearity voltage. For $\alpha=$ 9.05, a period-4 solution is obtained, as shown in Fig. 15(a). By increasing $\alpha$, the collision between the LC and saddle EP $E P_{2}=0 \mathrm{~V}$ takes place for $\alpha=9.09$, as shown in Fig. 15(b). This gives rise to the chaotic attractor.

When considering also the $\beta$ variations, the bifurcation loci of Fig. 16 are obtained. Time-domain simulations are superim- posed. The locus providing the start-up of the frequency division by four is not represented since it is extremely close to the chaos-onset locus. However, for the determination of the collision between the LC and unstable EP $E P_{2}=0 \mathrm{~V}$, a period-4 steady-state solution is considered. As expected, the calculation of the realistic waveform significantly improves the chaos prediction in comparison with previous results where period doublings were neglected [15]. This is clearly shown in the loci expanded view of Fig. 17. Note that an excellent correspondence is obtained between the time-domain simulations and the homoclinic orbit prediction from a period-4 waveform.

\section{CONCLuSiON}

In this paper, a new method is proposed for the detection of the onset of chaos through the HB technique. Thus, this method is well suited for microwave frequencies where time-domain techniques are often impractical. In addition, the new method makes use of commercial HB software, with its advantages of great flexibility and easy utilization by microwave-circuit designers. A new technique is presented for the prediction and steady-state analysis of successive frequency divisions by two in commercial software, which improves the accuracy for the onset of chaos calculation in the homoclinic routes and also enables the chaos prediction in the common period-doubling routes. The new perturbation technique introduced here opens exciting possibilities for the stability analysis of microwave circuits by using commercial software. Three circuits are analyzed by means of the new method: two varactor-based circuits, with a period-doubling route, and the classical Chua's circuit, exhibiting homoclinic chaos. In both cases, an excellent agreement is found with time-domain simulations.

\section{REFERENCES}

[1] A. Suárez, J. Morales, and R. Quéré, "Chaos prediction in a MMIC frequency divider in millimetric band," IEEE Microwave Guided Wave Lett., vol. 8, pp. 21-23, Jan. 1998.

[2] L. M. Pecora and T. L. Carroll, "Synchronization in chaotic systems," Phys. Rev. Lett., vol. 64, pp. 821-824, 1990.

[3] C. P. Silva and A. M. Young, "Implementing RF broad-band chaotic oscillators: Design issues and results," in Proc. IEEE Int. Circuits Syst. Symp., Monterey, CA, May 1998, pp. 489-493.

[4] C. P. Silva, "Shil'nikov theorem-A tutorial," IEEE Trans. Circuits Syst., vol. 40, pp. 675-682, Oct. 1993.

[5] J. Guckenheimer and P. Holmes, Nonlinear Oscillations, Dynamical Systems and Bifurcations of Vector Fields, 3rd ed. Berlin, Germany: Springer-Verlag, 1990.

[6] S. Wiggins, Introduction to Applied Nonlinear Dynamical Systems and Chaos. Berlin, Germany: Springer-Verlag, 1990.

[7] R. Genesio and A. Tesi, "A harmonic balance approach for chaos prediction: Chua's circuit," Int. J. Bifurcation Chaos, vol. 2, no. 1, pp. 61-79, Jan. 1992.

[8] M. Odyniec, "Nonlinear synchronized LC oscillators: Theory and simulation," IEEE Trans. Microwave Theory Tech., vol. 41, pp. 774-780, May 1993.

[9] R. Quéré, E. Ngoya, M. Camiade, A. Suárez, M. Hessane, and J. Obregón, "Large signal design of broad-band monolithic frequency dividers and phase-locked oscillators," IEEE Trans. Microwave Theory Tech., vol. 41, pp. 1928-1938, Nov. 1993.

[10] T. Matsumoto, "Chaos in electronic circuits," IEEE Trans. Circuits Syst. (Special Issue on Chaotic Systems), vol. 75, pp. 1033-1057, Aug. 1987.

[11] V. Rizzoli and A. Lipparini, "General stability analysis of periodic steady state regimes in nonlinear microwave circuits," IEEE Trans. Microwave Theory Tech., vol. MTT-33, pp. 30-37, Jan. 1985. 
[12] V. Rizzoli and A. Neri, "State of the art and present trends in nonlinear microwave CAD techniques," IEEE Trans. Microwave Theory Tech., vol. 36, pp. 343-356, Feb. 1988.

[13] M. J. Hasler, "Electrical circuits with chaotic behavior," IEEE Trans. Circuits Syst. (Special Issue on Chaotic Systems), vol. 75, pp. 1009-1021, Aug. 1987.

[14] S. Basu, S. M. Maas, and T. Itoh, "Stability analysis for large signal design of a microwave frequency doubler," IEEE Trans. Microwave Theory Tech., vol. 43, pp. 2890-2898, Dec. 1995.

[15] A. Suárez and J. M. Collantes, "Chaos detection in microwave circuits using harmonic balance commercial simulators," in IEEE MTT-S Int Microwave Symp. Dig., Baltimore, MD, June 1998, pp. 271-274.

[16] V. Iglesias, A. Suárez, and J. L. García, "New technique for the determination through commercial software of the stable-operation parameter ranges in nonlinear microwave circuits," IEEE Microwave Guided Wave Lett., vol. 8, pp. 424-426, Dec. 1998.
Juan-Mari Collantes (M'98) was born in Bilbao, Spain, in 1966. He received the degree in electronic physics from the University of the Basque Country, Bilbao, Spain, in 1990, the Ph.D. degree in electronics from the University of Limoges, Limoges, France in 1996, and the Ph.D. degree in electronics from the University of Cantabria, Santander, Spain in 1997.

From July 1996 to December 1996, and from July 1998 to December 1998, he was with the Hewlett-Packard Company, Santa Rosa, CA, as an Invited Researcher. Since February 1996, he has been an Associate Professor in the Electricity and Electronics Department, University of the Basque Country. His areas of interest include nonlinear design of microwave circuits, nonlinear modeling of microwave devices, and the investigation of chaotic regimes.

Almudena Suárez (M'96) was born in Santander, Spain, in 1964. She received the electronic physics degree and Ph.D. degree from the University of Cantabria, Santander, Spain, in 1987 and 1992, respectively, and the Ph.D. degree in electronics from the University of Limoges, Limoges, France, in 1993.

In 1987, she joined the Electronics Department, University of Cantabria, where she was involved with nonlinear simulation. From May 1990 to December 1992, she was on leave at the Laboratory IRCOM, University of Limoges. Since 1995, she has been an Associate Professor at the University of Cantabria, and a member of its Communications Engineering Department. Her areas of interest include the nonlinear design of microwave circuits and especially the nonlinear stability analysis and the investigation of chaotic regimes. 